\title{
Controle do escurecimento enzimático e da firmeza de polpa em pêssegos minimamente processados
}

\author{
Control of enzymatic browning and flesh firmness in fresh-cut peaches

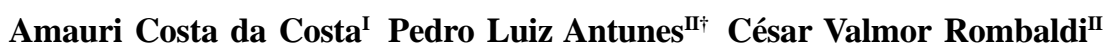 \\ Márcia Arocha Gularte ${ }^{\mathrm{III}}$
}

\section{RESUMO}

\begin{abstract}
O escurecimento enzimático e o amolecimento são as principais alterações que podem afetar pêssegos minimamente processados (MP). Objetivou-se avaliar a aplicação de L-cisteína (Cis), ácido L-ascórbico (AA) e cloreto de cálcio (CC) no controle do escurecimento enzimático e da perda de firmeza de polpa de pêssegos MP, cv. 'Esmeralda'. Os frutos foram processados e, após o fatiamento, imersos nos tratamentos: (I) água (controle); (II) $A A+C C$; (III) Cis $+C C$; e, (IV) Cis $+A A+C C$. Em seguida, as fatias foram acondicionadas em embalagens rígidas envoltas com filme PVC de $30 \mu m$ de espessura e armazenadas durante seis dias a $4 \pm 1^{\circ} \mathrm{C}$ e $85 \pm 3 \%$ UR. As amostras foram avaliadas quanto aos aspectos físicoquímicos, enzimáticos e sensoriais a cada dois dias. $O$ tratamento $C i s+A A+C C$ resultou em maiores valores de $L^{*} e$ $h^{o}$, o que indica ter sido o mais eficaz na prevenção do escurecimento. Os tratamentos com L-cisteina resultaram em menor atividade da polifenoloxidase (PPO) e o tratamento $C i s+A A+C C$ foi o mais efetivo na contenção da atividade da peroxidase (POD), indicando que o melhor desempenho desse tratamento está associado à menor atividade de PPO e POD. $A$ avaliação sensorial indicou que o tratamento $C i s+A A+C C$ foi o mais eficiente, proporcionando a manutenção de características sensoriais aceitáveis aos pêssegos MP, cv. 'Esmeralda'.
\end{abstract}

Palavras-chave: Prunus persica, processamento mínimo, antioxidantes, cisteína, ácido ascórbico, cloreto de cálcio.

\section{ABSTRACT}

The enzymatic browning and firmness loss are the major changes that could affect fresh-cut peaches. The objective

\begin{abstract}
was to evaluate the application of L-cysteine (Cis), L-ascorbic acid (AA) and calcium chloride (CC) in the control of browning enzymatic and loss of firmness of minimally processed dried peaches, $c v$. 'Esmeralda'. The fruits were processed industrially and, after slicing, immersed in treatments: (I) water (control); (II) AA+CC; (III) Cis $+C C$ and (IV) Cis $+A A+C C$. After, the slices were packed in packages sealed with PVC film $30 \mu \mathrm{m}$ thick and stored for six days at $4 \pm 1^{\circ} \mathrm{C}$ and $85 \pm 3 \%$ UR. The samples were evaluated for their physical-chemical, enzymatic and sensory aspects every two days. Treatment $C i s+A A+C C$ resulted in higher values of $L^{*}$ and $h^{\circ}$, which indicates that it was the most effective in browning prevention. Treatments with L-cysteine resulted in lower activity of polyphenoloxidase (PPO) and treatment $C i s+A A+C C$ was the most effective in containing peroxidase (POD) activity, indicating that the best performance for such treatment is associated with lower activity of PPO and POD. The sensory evaluation indicated that the treatment $C i s+A A+C C$ was the most efficient, providing the maintenance of the sensory characteristics acceptable for fresh-cut peaches, $c v$. 'Esmeralda'.
\end{abstract}

Key words: Prunus persica, minimal processing, antioxidants, cysteine, ascorbic acid, calcium chloride.

\section{INTRODUÇÃO}

Os pêssegos minimamente processados (MP) são produtos altamente perecíveis, com metabolismo respiratório e de maturação mais ativos do que em frutos não processados. Além disso, a maioria das cultivares de pêssego é altamente sensível

Instituto Federal Sul-Riograndense, Campus Pelotas Visconde da Graça. Programa de Pós-graduação em Ciência e Tecnologia Agroindustrial, Universidade Federal de Pelotas (UFPel). Av. Ildefonso Simões Lopes, 2791, 96087-000, Pelotas, RS, Brasil. Email: amauri.costa@ufpel.edu.br. Autor para correspondência.

IIDepartamento em Ciência e Tecnologia Agroindustrial, Faculdade de Agronomia Eliseu Maciel (FAEM), UFPel, Pelotas, RS, Brasil.

IIIDepartamento de Ciência dos Alimentos, Faculdade de Administração e Turismo, UFPel, Pelotas, RS, Brasil. 
ao escurecimento enzimático, seja pela elevada concentração de compostos fenólicos e/ou atividade de enzimas oxidativas, com destaque para polifenoloxidases (PPO) e peroxidases (POD) (GIRNER et al., 2002).

Dentre as medidas comumente utilizadas para prevenir essas alterações, estão o uso de antioxidantes, como ácidos ascórbico/cítrico, aminoácidos e de protetores da textura, como sais de cálcio (GORNY etal., 1999; MOLINE et al., 1999; VILASBOAS et al., 2001; ELEZ-MARTÍNEZ et al., 2005; ROJAS-GRAÜ et al., 2006; RAYBAUDI-MASSILIA et al., 2007). O ácido L-ascórbico e seus vários sais neutros constituem-se nos principais antioxidantes para o uso em frutos, hortaliças e sucos, visando a prevenir o escurecimento e outras reações oxidativas (SAPERS et al., 1993). Aminoácidos que contêm enxofre, bem como seus derivados, foram estudados como inibidores do escurecimento enzimático (GORNY et al., 2002). É o caso da L-cisteína, que contém um grupo tiol, capaz de reduzir $\theta$-quinonas em $\theta$-difenóis, impedindo a formação de pigmentos escuros ou reagindo $\operatorname{com} \theta$-quinonas para produzir compostos incolores (RICHARD-FORGET et al., 1992). Sais de cálcio, particularmente o cloreto e o lactato, têm sido utilizados, geralmente em combinação com agentes antioxidantes, na prevenção do amaciamento de vegetais minimamente processados (GORNY et al., 1999; RAYBAUDI-MASSILIA et al., 2007).

Baseando-se no fato de que a L-cisteína e o ácido L-ascórbico atuam tanto como moléculas antioxidantes quanto no controle da atividade das enzimas PPO e POD, e que o cálcio pode contribuir para a preservação da firmeza da polpa, acredita-se que a combinação desses compostos possa prevenir o escurecimento enzimático, bem como preservar a textura de pêssegos da cultivar 'Esmeralda'.

\section{MATERIAL E MÉTODOS}

Utilizaram-se pêssegos, cv. 'Esmeralda', safra 2008, provenientes de pomar comercial situado no município de Morro Redondo, RS (3135'S-52³7'O), colhidos no estádio de maturação plena, imediatamente transportados para a Indústria Piloto do Conjunto Agrotécnico "Visconde da Graça", da Universidade Federal de Pelotas, e armazenados em câmara fria por $24 \mathrm{~h}$ a $1 \pm 1^{\circ} \mathrm{C}$ e $80-85 \%$ de U.R. Os frutos foram classificados em função do diâmetro (mínimo 6,0 cm); selecionados em função do estádio de maturação; descaroçados, utilizando um descaroçador industrial por torção; descascados com pelador tipo cascata, mediante aspersão de lixívia $(\mathrm{NaOH}$ a $10 \% \mathrm{~m} / \mathrm{v})$ a $95^{\circ} \mathrm{C}$, com tempo de retenção de cerca de $3 \mathrm{~min}$; lavagem com água à temperatura ambiente; submetidos a uma nova seleção, retoque e corte de cada metade em quatro partes no sentido longitudinal. As fatias foram imersas por $5 \mathrm{~min}$ nas soluções contendo os seguintes tratamentos: (I) água destilada (controle); (II) ácido Lascórbico $1 \%(\mathrm{~m} / \mathrm{v})+$ cloreto de cálcio $1 \%(\mathrm{~m} / \mathrm{v}) ;$ (III) cloridrato de L-cisteína $1,5 \%(\mathrm{~m} / \mathrm{v})+$ cloreto de cálcio $1 \%(\mathrm{~m} / \mathrm{v})$ e (IV) cloridrato de L-cisteína 1,5\% (m/v) + ácido ascórbico $1 \%(\mathrm{~m} / \mathrm{v})+$ cloreto de cálcio $1 \%(\mathrm{~m} / \mathrm{v})$. $\mathrm{O}$ excesso de líquido foi drenado e unidades experimentais contendo $200 \mathrm{~g}$ de fatias de pêssego foram acondicionadas em bandejas rígidas de polipropileno $\left(11,5\right.$ x 8,0 x 4,0cm - Galvanotek ${ }^{\circledast}$ - Ref. G-88), as quais foram recobertas por filme de PVC de $30 \mu \mathrm{m}$ de espessura e imediatamente armazenadas em câmara fria, durante seis dias, com controle de temperatura $\left(4 \pm 1^{\circ} \mathrm{C}\right)$ e umidade relativa $(85 \pm 3 \% \mathrm{UR})$. Em cada repetição de cada tratamento, em cada coleta, foram realizadas três análises com o experimento sendo conduzido em delineamento inteiramente casualizado.

Para monitorar a conservação dos pêssegos durante o armazenamento, avaliou-se o $\mathrm{pH}$, a acidez total titulável (ATT) e o teor de sólidos solúveis totais (SST). Para testar-se a hipótese, selecionaram-se como variáveis dependentes, a firmeza, a luminosidade $\left(L^{*}\right)$, o ângulo de cor $\left(h^{\circ}\right)$, a atividade enzimática (PPO e POD) e a avaliação sensorial do produto. AATT, o pH e o teor de SST foram determinados seguindo o método descrito por PEGORARO et al. (2010). Para a determinação da cor, foi utilizado colorímetro "Minolta", modelo CR-300, com leituras das coordenadas $L^{*}, a^{*} \mathrm{e}$ $b^{*}$. A avaliação de firmeza foi realizada por determinação da resistência à penetração usando texturômetro Texture Analyser ${ }^{\circledR}$, modelo TA. XT. PLUS (Stable Micro Systems Ltd., Surrey, Inglaterra) com célula de carga de $5 \mathrm{~kg}$. O diâmetro da ponta de teste utilizada foi de $2 \mathrm{~mm}$, as velocidades de pré-teste, teste e pós-teste de $2 \mathrm{~mm} \mathrm{~s}^{-1}, 2 \mathrm{~mm} \mathrm{~s}^{-1}$ e $10 \mathrm{~mm} \mathrm{~s}^{-1}$, respectivamente, e distância de penetração de $30 \mathrm{~mm}$. Os resultados foram expressos em Newton (N).

As determinações das atividades da PPO e POD seguiram o método adaptado por TORALLES et al., (2004) a partir do método descrito por FLURKEY \& JEN (1978). Os resultados foram expressos como atividade enzimática capaz de alterar 0,01 de absorbância por grama de polpa fresca por minuto $\left(\mathrm{UA} \cdot \mathrm{g}^{-1} \cdot \mathrm{min}^{-1}\right.$ ).

$\mathrm{Na}$ avaliação sensorial, foi utilizado um método descritivo através do teste de Avaliação de Atributos (FARIA et al., 2000), com equipe de quinze julgadores previamente treinados por três semanas (testes discriminativos triangular e duo-trio, e após o teste descritivo avaliação de atributos), que avaliaram 
as características de cor, doçura, acidez e consistência, utilizando escala não estruturada de $9 \mathrm{~cm}$, cujo extremo esquerdo corresponde à menor intensidade $\mathrm{e} o$ direito, à maior intensidade. Os resultados foram analisados por ANOVA e de comparação das médias através do teste de Tukey $(p \leq 0,5)$. O programa estatístico utilizado foi o Statistical Analysis System for Windows V8.

\section{RESULTADOS E DISCUSSÃO}

Avaliações físico-químicas

Os valores de $\mathrm{pH}$ (Figura 1A), ATT (Figura

1B) e SST (Figura 1C) observados após o processamento são coerentes com pêssegos da cultivar 'Esmeralda', caracterizada como de ciclo médio (TORALLES et al., 2004), ou seja, pH entre 2,31 e 3,49; ATT de 0,86 a $1,13 \%$ de ácido cítrico e SST de 8,0 a $11,0^{\circ}$ Brix. Esses valores são similares à maioria das cultivares de pêssego estudadas no processamento mínimo, seja para consumo como única fruta ou na composição de saladas de frutas (ZHU, et al., 2009; CRISOSTO \& CRISOSTO, 2003; GORNY et al., 1999), nas quais se busca, além do sabor típico da fruta, uma relação SST/ATT entre 10 e 20 (MEREDITH et al., 2009).

Durante os dois primeiros dias de armazenamento, houve redução do $\mathrm{pH}$, havendo estabilização dessa variável entre o segundo e sexto dia de avaliação. Além disso, nos tratamentos com $\mathrm{Cis}+\mathrm{AA}+\mathrm{CC}$ e $\mathrm{Cis}+\mathrm{CC}$ os valores foram significativamente menores do que nos demais tratamentos, provavelmente devido à dissociação da L-cisteína, tendo em vista seu valor da constante de dissociação $\left(\mathrm{pK}_{\mathrm{a}}\right)$ ser de 1,96. Na presença do ácido ascórbico, não deve ter ocorrido a dissociação em virtude de seu valor de $\mathrm{pK}_{\mathrm{a}}$ ser superior $(4,17)$ aos valores de $\mathrm{pH}$ do tratamento controle, entre 3,49 e 2,75.

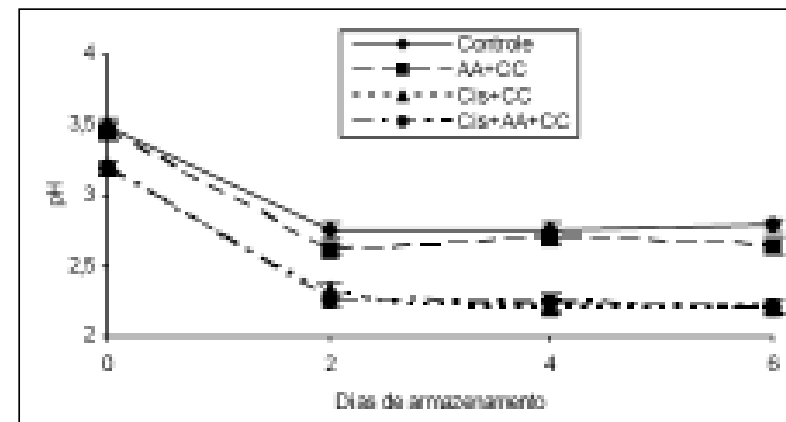

[a)

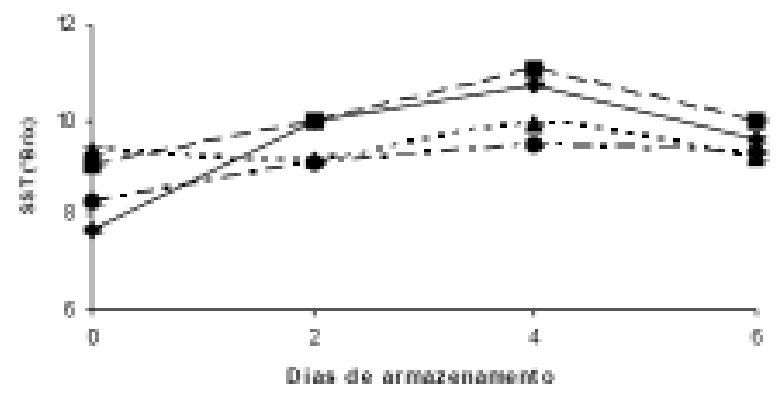

(c)

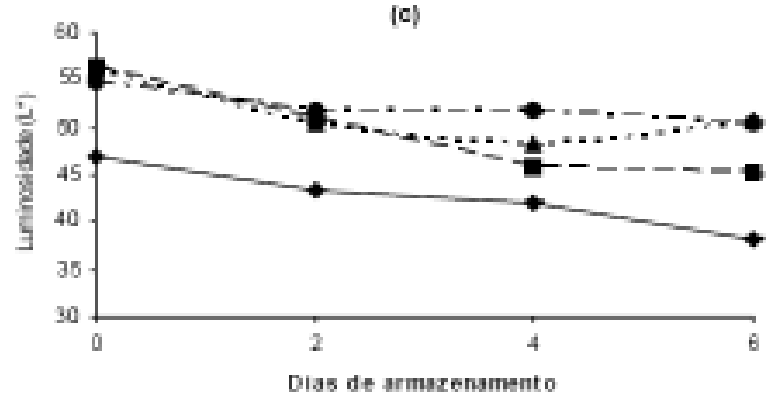

(e)

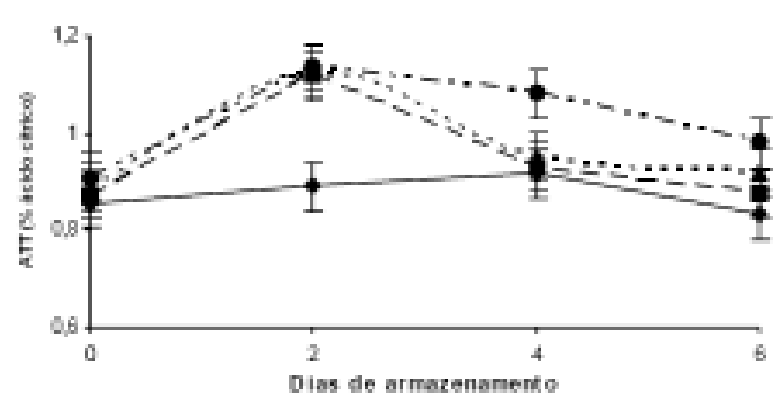

(b)

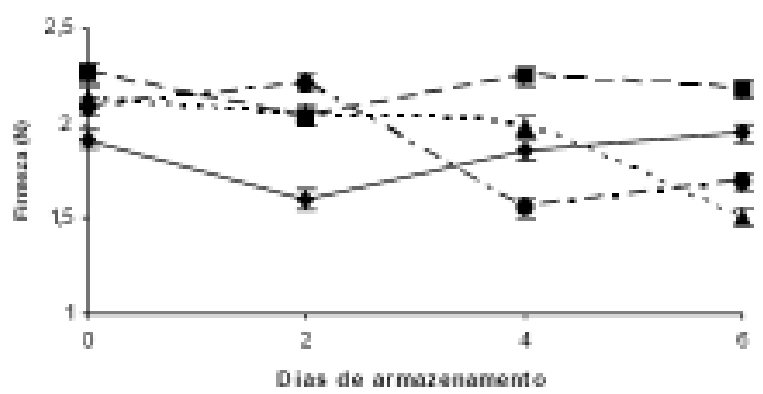

(d)

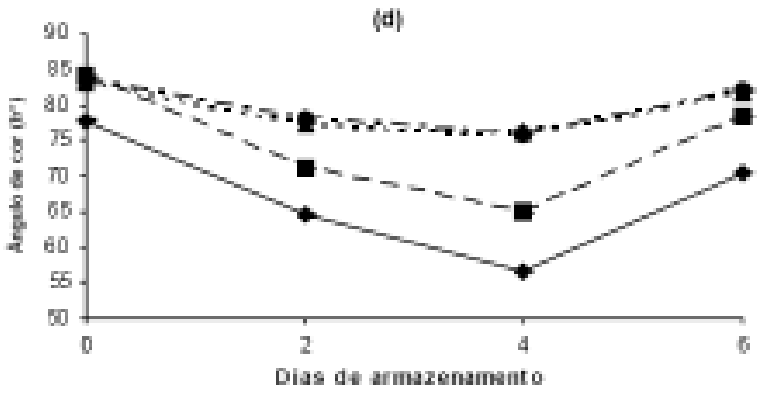

(1)

Figura 1 - Caracterização físico-química (pH, ATT, SST, Firmeza, Luminosidade e Ângulo de cor) de pêssegos, cv. 'Esmeralda', minimamente processados e submetidos a diferentes tratamentos, armazenados a $4^{\circ} \mathrm{C}$ e $85 \pm 3 \%$ UR. 
A ocorrência de reduções nos valores de $\mathrm{pH}$ nas primeiras $24 \mathrm{a} 48 \mathrm{~h}$ após o processamento mínimo de frutos é normal e decorrente do aumento da dissociação de ácidos orgânicos (GOMES et al., 2010; ZHU et al. 2009). Complementarmente, segundo GOMES et al. (2010), o aumento da concentração hidrogeniônica pode contribuir para uma melhor preservação das características físico-químicas de frutos minimamente processados.

$\mathrm{O}$ tratamento $\mathrm{AA}+\mathrm{CC}$ foi o que melhor atuou na preservação da firmeza de polpa (Figura 1D), o que está de acordo com GORNY et al. (1999), segundo os quais, a combinação de ácido ascórbico $2 \%(\mathrm{~m} / \mathrm{v})+$ lactato de cálcio $1 \%(\mathrm{~m} / \mathrm{v})$ evitou a redução de firmeza em fatias de pêssego, cv. 'Carnival'. De modo geral, o uso de cloreto de cálcio contribui para a preservação desse atributo, como foi demonstrado por MIGUEL et al. (2007) em melancias minimamente processadas. Porém tem sido observado que as respostas à aplicação desse sal de cálcio podem ser significativamente potencializadas ou reduzidas pelo uso de tratamentos complementares. Por exemplo, SOLIVA-FORTUNY et al. (2006) observaram que a combinação de ácido ascórbico e cloreto de cálcio não contribuiu para uma melhor preservação de maçãs minimamente processadas, diferentemente do que foi observado por GORNY et al. (1999) em pêssegos.

A diminuição da firmeza de polpa, observada no tratamento $\mathrm{Cis}+\mathrm{CC}$ nos últimos dias de avaliação e tratamento $\mathrm{Cis}+\mathrm{AA}+\mathrm{CC}$ entre o segundo e quarto dias, pode ser consequência do processamento mínimo e do processo de senescência, visto que esse tipo de processamento aumenta a perecibilidade do produto, pelo aumento da atividade metabólica e pela descompartimentalização de enzimas e substratos, que não puderam ser evitadas eficazmente, ainda que pela utilização do cloreto de cálcio. Isto pode também ser decorrente do fato de que a solubilização da parede celular é uma consequência da ação de enzimas que necessitam de condições com baixo potencial de oxidação (PEGORARO et al., 2010), o que pode ter sido proporcionado pela L-cisteína, que atua como agente redutor (SAPERS, 1993). Desse modo, a L-cisteína pode ter atuado como indutora do metabolismo de hidrólise das macromoléculas da parede celular.

No que concerne às variações de coloração, detectou-se redução no valor $L^{*}$ dos frutos (Figura $1 \mathrm{E})$, independente do tratamento, indicando o gradativo escurecimento ao longo do armazenamento. Os menores valores de $L^{*}$ foram observados no tratamento controle, o que corrobora com o fato de serem frutos com tendência ao escurecimento. Esses resultados assemelham-se aos de MOLINE et al. (1999) ao verificarem que a combinação de ácido cítrico $\left(0,5 \mathrm{~mol} \cdot \mathrm{L}^{-1}\right)$ e $\mathrm{N}$-acetilcisteína $\left(0,05 \mathrm{~mol} . \mathrm{L}^{-1}\right)$ foi altamente eficaz na prevenção do escurecimento em bananas, assim como MELO \& VILAS-BOAS (2006), ao concluírem que o tratamento com ácido ascórbico $1 \%(\mathrm{~m} / \mathrm{v})+$ cloreto de cálcio $1 \%(\mathrm{~m} / \mathrm{v})+$ cisteína $1,5 \%(\mathrm{~m} / \mathrm{v})$ foi o mais efetivo na prevenção das modificações de coloração em banana maçã. Da mesma forma, RAYBAUDIMASSILIA et al. (2007) encontraram valores de $L^{*}$ maiores para o tratamento com N-acetil-L-cisteína $1 \%$ $(\mathrm{m} / \mathrm{v})+$ glutationa $1 \%(\mathrm{~m} / \mathrm{v})+$ lactato de cálcio $1 \%(\mathrm{~m} /$ v) em comparação com o mesmo valor adicionado de ácido málico 2,5\% (m/v) na prevenção do escurecimento em maçãs, cv. 'Fuji', minimamente processadas. ROJAS-GRAÜ et al. (2006) encontraram resultados semelhantes ao avaliar a eficácia de diferentes compostos na prevenção do escurecimento em maçã, indicando que $\mathrm{N}$-acetil-L-cisteína e glutationa são os mais eficazes. Desse modo, verificou-se que o uso da L-cisteína contribuiu para prevenir o escurecimento (Figura 1E), mas não se constitui na melhor alternativa para a prevenção da firmeza de polpa (Figura 1D).

O uso de ácido ascórbico associado ao cloreto de cálcio não foi capaz de prevenir o escurecimento a partir do segundo dia de armazenamento. Isso indica que ele, embora seja um bom antioxidante (SAPERS et al., 1993), é capaz de evitar a alteração de coloração dos frutos apenas por períodos relativamente curtos (dois dias).

À semelhança do que foi observado quando da avaliação da variável $L^{*}$, o ângulo de cor $\left(h^{\circ}\right)$ também foi menor em pêssegos do tratamento controle e naqueles com CC+AA (Figura 1F). Contrastando com esses tratamentos, os maiores valores de $h^{o}$, em média $90^{\circ}$, o que representa uma coloração amarelo-alaranjada, foram detectados em frutos nos quais a L-cisteína estava presente na solução de tratamento. Mais uma vez, há a demonstração da ação desse aminoácido como meio de prevenção ao escurecimento de pêssegos cv. 'Esmeralda'.

\section{Avaliações enzimáticas}

Quando se avaliou a atividade da PPO (Figura 2A), verificou-se que os valores foram superiores a $0,002 \mathrm{UA} \mathrm{min}^{-1} \mathrm{~g}^{-1}$, nos frutos não tratados. Essas atividades são elevadas, baseando-se no trabalho de LEE et al. (1990), que afirmam que valores acima de $0,0005 \mathrm{UA} \mathrm{min}^{-1} \mathrm{~g}^{-1}$ indicam cultivares com suscetibilidade ao escurecimento, tendo em vista que, em geral, não há limitação de substrato em pêssegos. A atividade da PPO foi significativamente reduzida pela aplicação de $\mathrm{Cis}+\mathrm{AA}+\mathrm{CC}, \mathrm{Cis}+\mathrm{CC}$ e AA+CC, indicando 


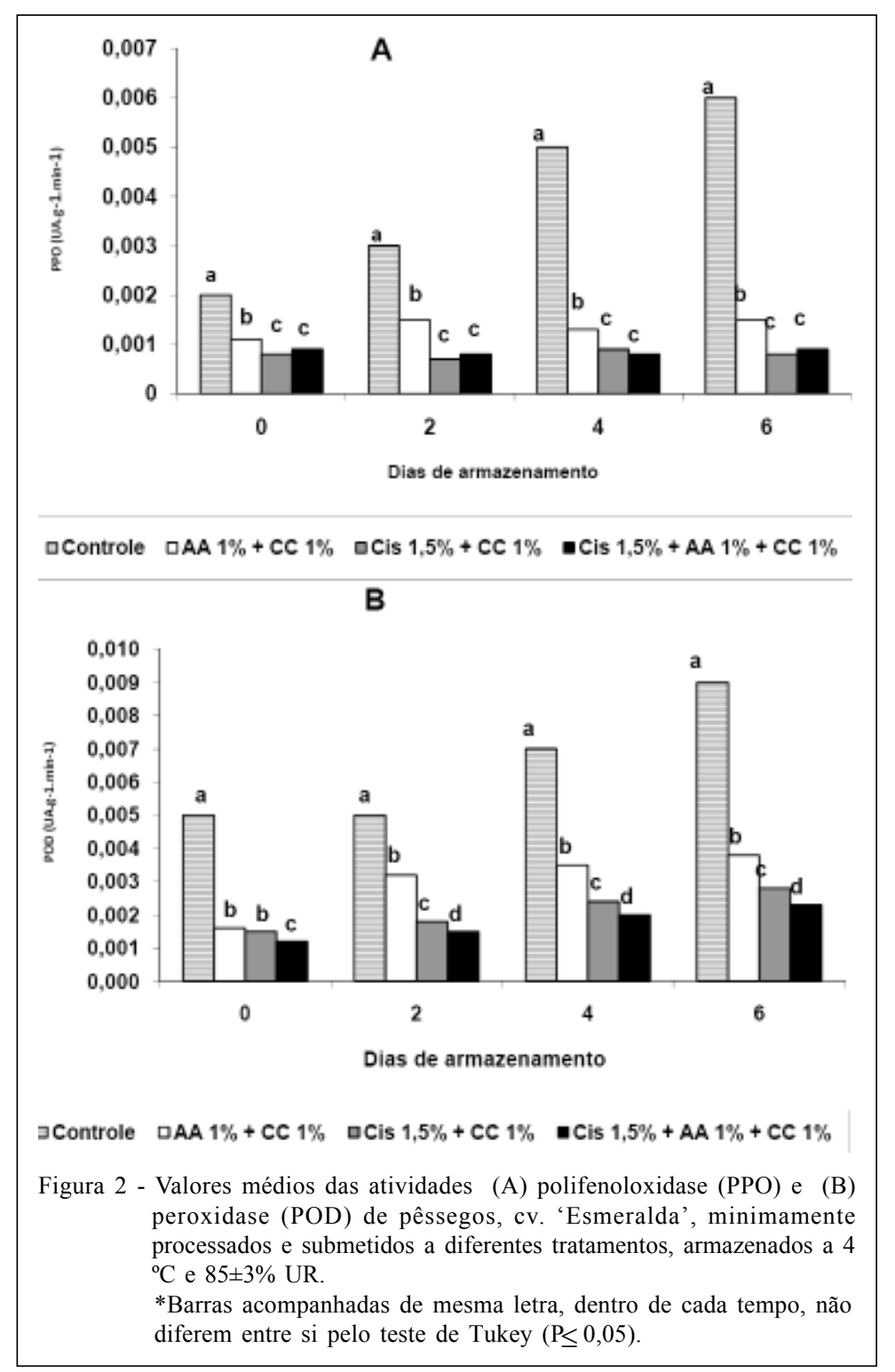

que esses compostos não apenas atuam como antioxidantes, mas também sobre a atividade dessa enzima. Tal comportamento foi mantido durante todo o período de estudo. $\mathrm{O}$ fato de haver ação do ácido Lascórbico sobre a atividade enzimática já era esperado, pois LAMIKANRA \& WATSON (2001) demonstraram que a elevada concentração dessa molécula pode reduzir a disponibilidade de cofatores para essa enzima. No entanto, é amplamente conhecido (SAPERS et al., 1993) que o ácido L-ascórbico é uma molécula antioxidante altamente reativa, o que resulta em respostas rápidas em termos de proteção à oxidação (BARSAN et al., 2010). Porém, em produtos fisiológicos nos quais as condições de oxidação são mantidas por tempos prolongados, o ácido L-ascórbico pode não ser suficiente para prevenir o escurecimento químico e/ou químico-enzimático. Isso pode explicar, ao menos em parte, o escurecimento observado em pêssegos tratados com essa molécula após dois dias de armazenamento.

A inclusão de L-cisteína nos tratamentos reduziu ainda mais a atividade da PPO. Essa pode ser uma das razões do menor escurecimento em pêssegos tratados com esse aminoácido (Figura 1E).

No que concerne à atividade da POD (Figura 2B), houve tendência similar à observada para a PPO. A aplicação de L-cisteína, ácido L-ascórbico e cloreto de cálcio foi capaz de reduzir significativamente a atividade dessa enzima, obtendo-se a melhor resposta com a combinação das três moléculas, indicando que 
essa associação constitui-se em ação fundamental na redução das atividades da PPO e da POD, e também na prevenção do escurecimento, tendo em vista que foi com essa combinação que se manteve a coloração mais próxima à inicial (Figura 1E).

\section{Avaliação Sensorial}

No atributo cor, os julgadores atribuíram maiores notas aos pêssegos que receberam tratamento com L-cisteína (Figura 3), o que está coerente com os valores de $L^{*}$ e do ângulo de cor $\left(h^{\circ}\right)$ das amostras de pêssego.

Independentemente dos tratamentos, as notas atribuídas à doçura (entre 1,51 e 3,12) foram baixas. A causa provável é o relativamente baixo teor de SST desses frutos, entre 8,0 e 11,0Brix. Em geral, há maior aceitabilidade por pêssegos com SST na faixa de 12,5 a 15,0³rix (CRISOSTO \& CRISOSTO, 2003), ou frutos com uma relação SST/ATT acima de 15
(MEREDITH et al., 1989). Os pêssegos cv. 'Esmeralda' apresentaram, em média, uma relação SST/ATT de 10. Mesmo assim, trata-se de uma cultivar com muito boa aceitabilidade sensorial no contexto nacional (TORALLES et al., 2004).

À semelhança da percepção dos julgadores frente à doçura, a acidez também recebeu notas baixas. Os valores de ATT, entre 0,86 a 1,13\%, enquadram essa cultivar como medianamente ácida, o que explica o resultado da análise sensorial. Além disso, os resultados analíticos observados no tratamento $\mathrm{C}$ is $+\mathrm{AA}+\mathrm{CC}$ nesse atributo, vão ao encontro dos comentários espontâneos acerca de uma acidez mais pronunciada desse tratamento, em relação aos demais, no quarto e sexto dias de avaliação.

De modo geral, a avaliação sensorial dos pêssegos confirmou as observações feitas através das variáveis analíticas, ou seja, o tratamento dos frutos com L-cisteína, ácido L-ascórbico e cloreto de cálcio

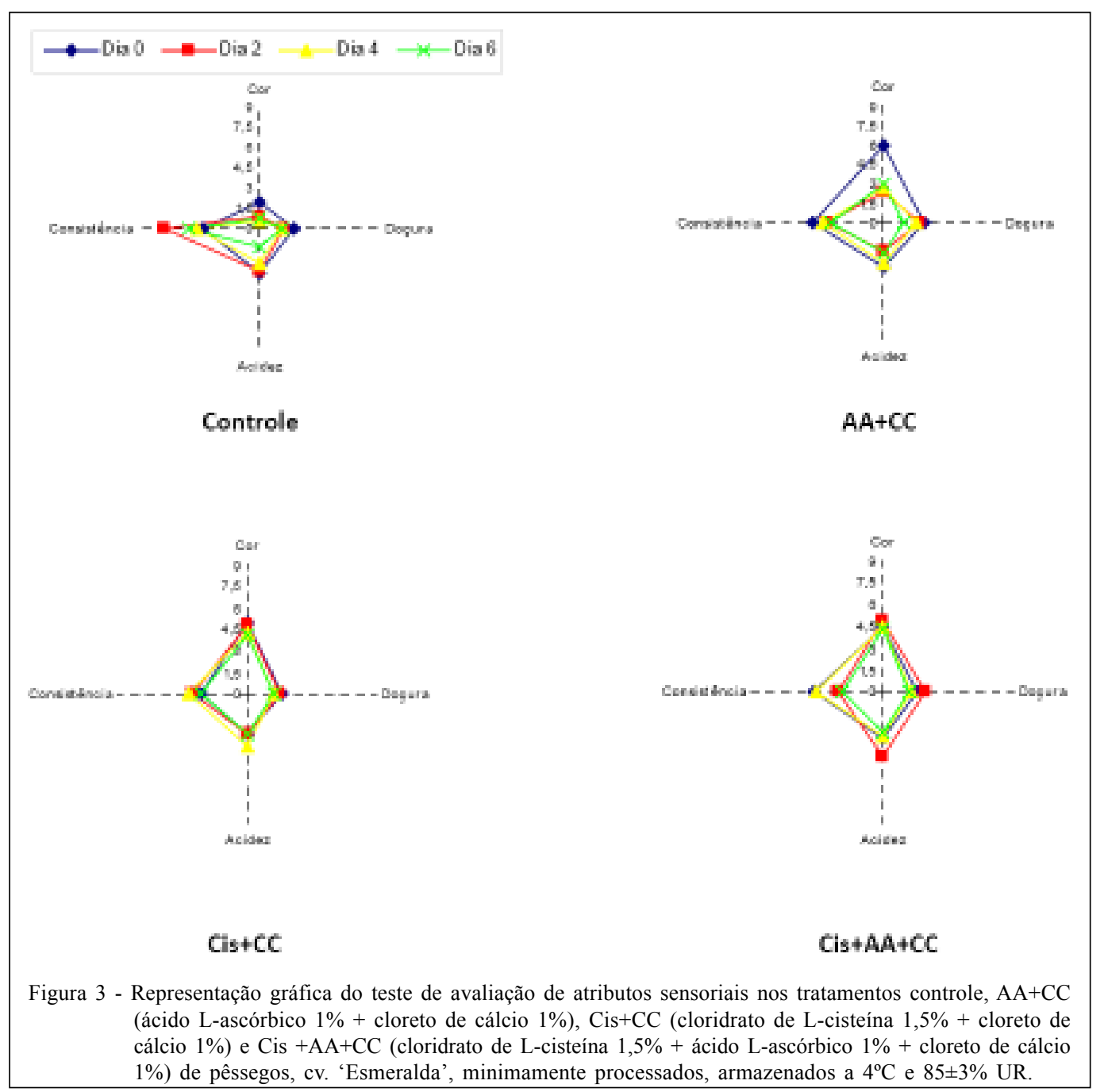

Ciência Rural, v.41, n.6, jun, 2011. 
resultou em melhor desempenho. Isso indica que, conforme detectado pelas variáveis $L^{*} \mathrm{e} h^{o}$, a melhor coloração foi detectada pelos julgadores nos frutos que receberam tratamento com L-cisteína. Por outro lado, a maior consistência foi evidenciada em pêssegos tratados com cloreto de cálcio.

\section{CONCLUSÃO}

O uso de L-cisteína e de cloreto de cálcio, associados ao ácido L-ascórbico, contribui para a manutenção da coloração e da textura de pêssegos 'Esmeralda' minimamente processados e refrigerados, por até seis dias.

\section{REFERÊNCIAS}

BARSAN, C. et al. Characteristics of the tomato chromoplast revealed by proteomic analysis. Journal of Experimental Botany, v.61, n.9, p.2413-2431. Disponível em: <http://jxb.oxfordjournals.org/content/61/9/ 2413 . a b stract ? s id $=8$ e $51516 \mathrm{c}-7789$ $428 \mathrm{c} 9 \mathrm{c} 1 \mathrm{be} 16750 \mathrm{a} 94 \mathrm{e} 5 \mathrm{c}>$. Acesso em: 28 set. 2010. doi: $10.1093 / \mathrm{j} \times \mathrm{b} / \mathrm{erq} 070$.

CRISOSTO, G.M.; CRISOSTO, C.H. Understanding consumer acceptance of peach, nectarine and plum cultivars. Acta Horticulturae, v.604, p.115-119, 2003. Disponível em: $<$ http://www.actahort.org/books/604/604_10.htm>. Acesso em: 17 abr. 2009.

ELEZ-MARTÍNEZ, P. et al. O. Natural Antioxidants Preserve the Lipid Oxidative Stability of Minimally Processed Avocado Purée. Journal of Food Science, v.70, n.5, p.325-329, 2005. Disponível: <http://onlinelibrary.wiley.com/doi/10.1111/ j.1365-2621.2005.tb09986>. Acesso em: 05 mai. 2009. doi: 10.1111/j.1365-2621.2005.tb09986.x.

FARIA, E.V. et al. Técnicas de análise sensorial. São Paulo: ITAL, 2000. 109p.

FLURKEY, W.; JEN, J. Peroxidase and Polyphenoloxidase actives in developing peaches. Journal of Food Science, v.43, n.6, p.1826-1831, 1978. Disponível em: <http:// onlinelibrary.wiley.com/doi/10.1111/j.13652621.1978.tb07424.x/>. Acesso em: 05 mai. 2009. doi: $10.1111 /$ j.1365-2621.1978.tb07424.x.

GIRNER, J., et al. Inactivation of peach polyphenoloxidase by exposure to pulsed electric fields. Journal of Food Science, v.67, n.4, p.264-267, 2002. Disponível em: <http:// onlinelibrary.wiley.com/doi/ $10.1111 /$ j. 1365 2621.2002.tb10307>. Acesso em: 01 ago. 2009. doi: 10.1111/ j.1365-2621.2002.tb10307.x.

GOMES, M.H, et al. Hidrogen íon concentration affects quality retention and modifies the effect of calcium additivies on fresh-cut 'Rocha' pear. Biology Postharvest and Technology, v.58, n.3, p.239-246, 2010. Disponível em: <http://www.sciencedirect.com/ science?_ob=ArticleListURL\&_method=list\&_ArticleListID $=$ $1713905509 \&$ s t $=13 \&_{-} \mathrm{sort}_{\mathrm{r}} \mathrm{r} \& \mathrm{sis} \mathrm{rterm}$ $=\&$ searchtype $=$ a\&originPage $=$ rslt_list\&_acct $=\mathrm{C} 0$
$00050221 \&$ \&ersion $=1 \&$ \& urlVersion $=0$ \&_userid $=$ $10 \& m d 5=d f 7 a 7320 \mathrm{~d} 622 \mathrm{e} 1549496039 \mathrm{cb} 6 \mathrm{be} 155 \mathrm{~d}>$. Acesso em: 23 set.2009. doi:10.1016/j. postharvbio.2010.07.004.

GORNY, J.R. et al. Quality changes in fresh-cut pear slices as affected by controlled atmospheres and chemical preservatives. Postharvest Biology and Technology, v.24, n.3, p.271-8. 2002. Disponível em: <http://www.sciencedirect.com/ science?_ob=ArticleListURL\&_method=list\&_ArticleListID $=1713932437 \&$ s o r t $=r \&$ s t $=13 \&$ vi e w $=$ c\&_acct $=\mathrm{C} 000050221 \&$ _version $=1 \&$ \&ur 1 Versio $\mathrm{n}=0 \& \quad$ u s e r i d $=10 \&$ m d $5=$ a 5 e 8 b 49 a b 7041 b 2f25a3934b7a6bdfd2\&searchtype=a>. Acesso em: 23 set. 2009. doi: 10.1016/S09255214(01)00139-9.

GORNY, J.R. et al. Quality Changes in Fresh-cut Peach and Nectarine Slices as Affected by Cultivar, Storage Atmosphere and Chemical Treatments. Journal of Food Science, v.64, n.3, p.429-432, 1999. Disponível em: <http://onlinelibrary.wiley.com/doi/10.1111/ j.1365-2621.1999.tb15057>. Acesso em: 28 mar. 2009. doi: 10.1111/j.1365-2621.1999.tb15057.x.

LAMIKANRA, O.; WATSON, M.A. Effects of ascorbic acid on peroxidase and polyphenoloxidase activities in fresh-cut Cantaloupe Melon. Journal of Food Science, v.66, n.9, p.1283 - 1286, 2001. Disponível em: <http://onlinelibrary.wiley.com/doi/10.1111/j.13652621.2001.tb15202>. Acesso em: 15 jul. 2009. doi: 10.1111/ j.1365-2621.2001.tb15202.x.

LEE, C.L. et al. Enzymatic browning in relation to phenolic compounds and polyphenoloxidase activity among various peach cultivars. Journal of Agricultural and Food Chemistry, v.38, p.99-101, 1990. Disponível em: <http:// pubs.acs.org/doi/abs/10.1021/jf00091a019>. Acesso em: 19 mai. 2009. doi: 10.1021/jf00091a019.

MELO, A.A.M.; VILAS BOAS, E.V.B. Inibição do escurecimento enzimático de banana maçã minimamente processada. Ciência e Tecnologia de Alimentos, v.26, n.1, p.110-115, 2006. Disponível em: <http://www.scielo.br/ scielo.php?script=sci arttext\&pid=S010120612006000100019\&lng $=$ en\&nrm=iso $>$. Acesso em: $07 \mathrm{abr}$. 2008. doi: 10.1590/S0101-20612006000100019.

MEREDITH, F.I. et al. Changes in physical and chemical parameters associated with quality and postharvest ripening of Harvester peaches. Journal of Agricultural and Food Chemistry, v.37, n.5, p.1210-1214, 1989. Disponível em: $<$ http://pubs.acs.org/doi/abs/10.1021/jf00089a002>. Acesso em: 18 ago. 2010. doi: 10.1021/jf00089a002.

MIGUEL, A.C.A. et al. Efeito do cloreto de cálcio na qualidade de melancias minimamente processadas. Horticultura Brasileira, v.25, n.3, p.442-446, 2007. Disponível em: $<$ http://www.scielo.br/scielo.php?script $=$ sci_arttext\&pid $=$ S01 $0205362007000300023 \& \operatorname{lng}=p t \& n r m=i s o>$. Acesso em: 07 maio 2008. doi: 10.1590/S0102-05362007000300023.

MOLINE, H.E. et al. Prevention of browning of banana slices using natural products and their derivatives. Journal of Food Quality, v.22, p.499-511, 1999. Disponível em: <http:// onlinelibrary.wiley.com/doi/ $10.1111 /$ j. 1745 4557.1999.tb00181>. Acesso em: 08 mai. 2008. doi: 10.1111/ j.1745-4557.1999.tb00181.x 
PEGORARO, C. et al. Physiological and molecular changes associated with prevention of woolliness in peach following preharvest application of gibberellic acid. Postharvest Biology and Technology, v.57, n.1, p.19-26, 2010. $<\mathrm{h} \mathrm{t} \mathrm{t} \mathrm{p} \mathrm{:} \mathrm{/} \mathrm{/} \mathrm{w} \mathrm{w} \mathrm{w.} \mathrm{s} \mathrm{c} \mathrm{i} \mathrm{e} \mathrm{n} \mathrm{c} \mathrm{e} \mathrm{d} \mathrm{i} \mathrm{r} \mathrm{e} \mathrm{c} \mathrm{t.} \mathrm{c} \mathrm{o} \mathrm{m} \mathrm{/}$ science? ob=ArticleListURL\&_method=list\&_ArticleListID $=1713$ $936685 \& \quad$ s ort $=r \& \quad s t=13 \&$ vi e w $=$ c \& a c c t $=$ 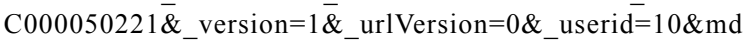 $5=170 \mathrm{~d} 1 \mathrm{fa} 8937837589 \mathrm{e} 4 \mathrm{~d} 4 \mathrm{dbad} 3407 \mathrm{~d} 84 \&$ searchtype $=\mathrm{a}>$. Acesso em: 22 set. 2010. doi: 10.1016/j. postharvbio.2010.02.003.

RAYBAUDI-MASSILIA, R.M. et al. Shelf-life extension of fresh-cut "Fuji" apples at different ripeness stages using natural substances. Postharvest Biology and Technology, v.45, n.2, p.265-275, 2007. <http://www.sciencedirect.com/ science?_ob=ArticleListURL\&_method $=1$ ist\&_ArticleListID $=171$ $3938195 \& \quad$ s o r t $=r \& \quad$ s t $=13$ \& vi e w $=\mathrm{c} \&$ 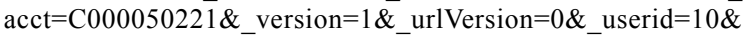 md5 $=$ e1be42039974b2599b31 ee 2 b769dd653\&searchtype $=a>$. Acesso em: 21 maio. 2009. doi: $10.1016 / \mathrm{j}$ postharvbio.2007.02.006.

RICHARD-FORGET, F.C. et al. Cysteine as an inhibitor of enzymatic browning. 2. kinetic studies. Journal of Agricultural and Food Chemistry, v.40, n.11, p.2.1082.113, 1992. Disponível em: <http://pubs.acs.org/doi/abs/ 10.1021/jf00023a014>. Acesso em: 05 nov. 2009. doi: 10.1021/ jf00023a014.
ROJAS-GRAÜ, M.A. et al. Browning Inhibition in Fresh-cut 'Fuji' Apple Slices by Natural Antibrowning Agents. Journal of Food Science, v.71, n.1, p.59-65, 2006. Disponível em: $<$ http://onlinelibrary.wiley.com/doi/10.1111/j.13652621.2006.tb12407.x/>. Acesso em: 21 out. 2009. doi: 10.1111/j.1365-2621.2006.tb12407.x

SAPERS, G.M. Browning of Food: Control by Sulfites, antioxidants and other means. Food Technology, v.47, n.10, p.75-84, 1993.

SOLIVA-FORTUNY, R.C. et al. Effects of Ripeness Stages on the Storage Atmosphere, Color, and Textural Properties of Minimally Processed Apple Slices. Journal of Food Science, v.67, n.5, p.1958-1963, 2006. Disponível em: <http:// onlinelibrary.wiley.com/doi/10.1111/j.13652621.2002.tb08752.x/>. Acesso em: 26 jul. 2008. doi: 10.1111/ j.1365-2621.2002.tb08752.x.

TORALLES, R.P. et al. Partial characterization of the enzymatic browning for polyphenoloxidase in peaches of the cv. Granada, Jade, Esmeralda and Maciel. Revista Brasileira de Agrociência, v.10, n.2, p.241-244, 2004.

ZHU, L.Q. et al. Inhibition of browning on the surface of peach slices by short-term exposure to nitric oxide and ascorbic acid. Food Chemistry, v.114, n.1, p.174-179, 2009. Disponível em: <http://www.sciencedirect.com/science/journal/03088>. Acesso em: 16 dez. 2010. doi: 10.1016/ j.foodchem.2008.09.036. 